\title{
EBF recommendation on the validation of bioanalytical methods for dried blood spots
}

Over the last few years bioanalysts, pharmacokineticists and clinical investigators have rediscovered the technique of dried blood spots. The revival has provided pharmaceutical R\&D a wealth of opportunities to optimize the drugdiscovery and development process with respect to animal and patient ethics, new scientific insights and costs savings. On the bioanalytical front, multiple experiments have been performed and a lot of experience has been gained. Nevertheless, the technique still has a number of bioanalytical challenges. The European Bioanalysis Forum discussed the advantages and hurdles of the technique and summarized their current thinking in a recommendation on the validation of bioanalytical methods for dried blood spots, which can be used as a cornerstone for further discussions and experiments.

The technique of dried blood spot (DBS) sampling has been used in neonatal screening since the early 1960s [1]. In recent years, the advantages of this technique have been recognized for use in therapeutic drug monitoring [2] and preclinical or clinical pharmacokinetic (PK) studies [3-7]. The advantages are multifold and relate to the ethical, practical, scientific and cost considerations. The most recognized ethical advantages are situated in the area of $3 \mathrm{R} s$ (refinement, reduction or removal of laboratory animals used for preclinical testing) and providing a more patient-friendly technique to sample blood in clinical studies. DBS sample collection typically requires less blood than conventional collection procedures and is a less invasive way (e.g., finger prick) to obtain required samples for patients and volunteers, especially children or newborns [8], thereby making it easy to include such PK assessments in clinical trials. In addition, the combination of less invasive sampling and removal of the need to harvest plasma or serum means that clinical studies are more easily executed in areas with no access to fully equipped clinical laboratories. Multiple references in literature highlight the potential use of DBS in the screening of biomarkers of tropical diseases $[9,10]$, opening a similar avenue of potential application in the area of phase 3 population PK in developing countries. The practical advantages for the clinical investigators, nurses and bioanalysts are equally important. By removing the need to store and ship samples on dry ice, the overall chain of custody is immensely simplified. Shipping and storage of the DBS samples on cards can occur at ambient temperature and through less complex routes. Moreover, there are significant scientific benefits as DBS can resolve persistent instability issues that result from conventional collection procedures [11,12]. As can be expected, all of the above advantages will positively impact the overall cost of the study conduct.

The increased use of DBS in regulated bioanalysis has stimulated the bioanalytical community to look in more detail into the validation requirements of DBS methods. Although overall acceptance criteria for method validation and study sample analysis will not be influenced because DBS samples are analyzed instead of liquid (i.e., plasma or serum) samples, it is important to recognize that the overall experimental protocol will need to be altered compared with the current paradigm of method development and validation in order to comply with the other scientific challenges posed by DBS. The European Bioanalysis Forum (EBF) has discussed in detail the currently used DBS methodology and experience. This paper is a summary of those internal discussions within the EBF, supplemented with input from EBF presentations at international meetings $[13,101,102]$ where we reached out to the bioanalytical community, and from literature [12]. The recommendations outlined are intended to provide guidance for method validation and analysis of DBS samples. Moving forward, these recommendations can serve as a cornerstone for future discussions, experiments and enhancement of the technique as more data are published in the scientific literature. This article
Philip Timmerman't, Steve White ${ }^{2}$, Susanne Globig ${ }^{3}$, Silke Lüdtke ${ }^{4}$, Leonarda Brunet ${ }^{5}$ \& John Smeraglia ${ }^{6}$

'Janssen Research \& Development, Bioanalysis Department, A Division of Janssen Pharmaceutica NV, Turnhoutseweg 30, B-2340,

Beerse, Belgium

${ }^{2}$ GlaxoSmithKline, UK

${ }^{3}$ Actelion Pharmaceuticals Ltd, Switzerland

${ }^{4}$ Boehringer-Ingelheim, Germany ${ }^{5} \mathrm{Abbott}$, France

${ }^{6}$ Pfizer, UK

†Author for correspondence:

Tel.: +32 |460 358|

E-mail: ptimmerm@its.jnj.com

Contributors, other EBF member companies listed at end of article.

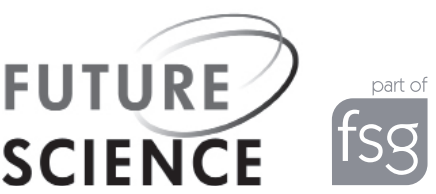


does not consider other dried matrix types, such as plasma and urine. It is possible that a number of the points discussed are relevant to these dried matrices, but it is also likely that there will be a number of differences when considering the analysis of these sample types.

\section{Introduction}

In our discussions, the EBF considers DBS to be part of the broader area of microsampling. As a consequence, we clearly want to make a distinction between both terminologies, with microsampling being an overarching terminology for experiments where significantly smaller samples (e.g., less than $50 \mu l$ ) are taken than normally applicable for a particular technique. As such, DBS may be classified within this category, if one of the aims of applying DBS is to lower the sampling volume. On the other hand, DBS may be applied to make use of additional advantages offered by the technique, without aiming at lowering the sampling volume per se, that is, solving a stability problem or facilitating Phase III clinical studies in developing countries. The recommendations provided in this article only reflect on (potential) differences and special considerations needed for method validation and sample analysis for DBS compared with traditional liquid assays. The recommendations are primarily given for 'spot and punch' techniques. Validation requirements for analysis via direct elution or direct analysis techniques may vary.

In this paper there is frequent reference to, or citation from, the US CDER/FDA guidance on bioanalysis $[14,15]$. Emerging guidance from other regions may reflect similarly on those topics. It is the EBF's strong belief that in the future the bioanalytical community will be able to refer to a unified guidance document, in line with the mission and vision of the Global Bioanalysis Consortium [103].

\section{EBF surveys}

Input was gathered from the EBF community, through surveys and iterations thereof. The iterations included targeted follow-up questions to understand in greater depth the potential hurdles. Information was also gathered from peer discussion at international scientific meetings. All information was summarized by an EBF-DBS subteam, consisting of the authors of this paper, and endorsed by the full EBF community. During 2009 and 2010, the EBF held several surveys gathering input from more than 25 companies to get a good understanding of current practices, opportunities and hurdles offered by DBS. In all discussions, the emphasis was on understanding the applicability of the current guidelines and the scientific translation thereof on bioanalytical method development when developing and validating methods for DBS analysis. In essence, the EBF community reflected on two major questions: do DBS methods require special validation criteria and if so, which validation criteria need to be included, deleted or adapted? Do studies in which the samples are analyzed with validated DBS methods need special acceptance criteria and if so, which acceptation criteria need to be altered? The scope of the questions included the sampling, sample handling, shipment and storage phases.

The outcome of both questions was surprising and clear at the same time. In essence, the EBF did not identify many experiments that were superfluous. On the other hand, the list of additional requirements or refinements was extensive.

Below, an overview is presented on the topics the EBF feels can be removed from bioanalytical method validation experiments for DBS methods, and of the experiments we recommend including or refining during bioanalytical method development and/or validation.

\section{General considerations \\ - Definition of a sample \& impact on inter- \& intra-card variability}

For liquid samples, the definition of a sample is well defined. However, some confusion can arise when splitting liquid samples at the clinical site or animal facility, in order to obtain a backup sample, for example, to allow aliquot $\mathrm{A}$ of a sample to be shipped to one laboratory for the analysis of compound $\mathrm{A}$ and aliquot $\mathrm{B}$ to a different laboratory for compound B, or to include additional experiments as stipulated in a (pre)clinical protocol. Nevertheless, in all of these cases, a backup sample B is considered to be identical to sample A, provided sample storage conditions are documented to be identical. Similarly, when the re-aliquoting of a sample for reanalysis is needed for different reasons such as technical error, PK repeat, metabolite quantification or incurred sample reanalysis (ISR), we consider the re-aliquoted sample to be identical to the original, provided the analysis is performed within the boundaries of the validated method.

When looking at the current sampling processes applied for DBS, there is limited similarity to liquid samples, hence the need for reflection 
and subsequent recommendations on how to define a sample. Typically, individual study samples are spotted in multiple replicates on one card. Nevertheless, it can occur that aliquots of individual study samples are spotted onto more than one card. The same holds true for individual calibration standards and quality control (Cal/QC) samples. Depending on laboratory procedures, they may be spotted in singles or replicates on one or multiple cards. In addition, preparation and spiking of $\mathrm{Cal} / \mathrm{QC}$ samples during validation may differ from the process used during study sample analysis with respect to replicates per card. As a consequence, intercard variability may impact accuracy and precision differences between study samples and $\mathrm{Cal} / \mathrm{QC}$ samples.

These sampling procedures in DBS require a clear understanding how we define 'one sample' and if we need to consider intra- versus inter-card variability as part of validation.

In practice, using current commercially available cards (four spots per card), Cal/QC samples will be spread over multiple cards as per the design of the validation experiments. This process is inherently part of the validation, therefore problems resulting from inter-card variability will become apparent in the variability of the data obtained, leading to the validation runs not meeting predefined acceptance criteria and be identified by subsequent root cause analysis. Similarly, during method application in clinical or preclinical studies, Cal/QC samples will be originating from multiple cards. Problems with inter-card reproducibility would again result in runs not meeting predefined acceptance criteria.

For the above reasons, the EBF recommends that, when using cards from the same type/manufacturer, inter-card variability does not need to be investigated as a discrete method validation parameter. In case a validation does not meet acceptance criteria, failed run investigation may focus on inter-card variability. In order to facilitate potential investigations, it is recommended to enable maximal visualization of inter-card variability as part of validation batch design.

The above reflections allow us to conclude that one single DBS can be considered to be one sample. Any additional spot originating from the identical liquid sample pool and spotted either on the same card or on a different card from the same type/manufacturer being an identical replicate sample, provided handling and storage are identical. This replicate spot can be used, for example, for reanalysis, metabolite quantification or ISR in analogy with re-aliquoting a liquid sample from the same sample tube or from a backup sample.

\section{Physical parameters of blood impacting the method validation}

Blood, having tissue-like properties, has significantly different physical behavior compared with liquid samples, which may impact sampleto-sample variability and assay robustness. The most important parameters affecting bioanalytical behavior are related to hematocrit variability, hemolysis and the potential use and impact of anticoagulants. Undue or nondocumented variations of these parameters can negatively influence spot formation and drying time, affecting homogeneity and size of the spot. Consequently, they should be investigated as part of the validation.

\section{Considerations of hematocrit}

Hematocrit is currently identified as the single most important parameter influencing the spread of blood on DBS cards, which could impact the validity of the results generated by DBS methods, affecting the spot formation, spot size, drying time, homogeneity and, ultimately, the robustness and reproducibility of the assays. This is particularly the case when a subsample punch is taken from the DBS, rather than punching the whole sample. Moreover, the scientific discussion of the ability to bridge blood concentrations with those for the same analyte in plasma/serum has resulted in greater experimental investigations and increased commentary $[16,17]$. This crosscomparison may be needed to compare plasma/ serum and blood PK, pharmacodynamic (PD) and toxicokinetic (TK) data across multiple studies, or fully understand the PK-PD or PK-TK relationship. With plasma being the matrix of choice for PK (or PD-TK) for decades, the scientific community is actively learning how to use blood concentration data in their evaluations. The PK, PD or TK challenges of blood concentrations are not part of this paper.

For bioanalytical method validation, the EBF recommends that the impact of variations of hematocrit values on the spot size and homogeneity should be understood and their impact on assay performance documented during validation. For that, clinically relevant variations of hematocrit values should be evaluated during validation (e.g., from $30-35$ to $55-60 \%$ ). Special populations, such as patients with physiological conditions or under medical treatment affecting the hematocrit beyond normal 
values (renal impairment, oncology patients), may require additional validation as they occur, such as including Cal and/or QCs to be prepared using matrix beyond normal hematocrit values.

As more data become available, the impact of hematocrit variability will need further discussion in the scientific community, both within bioanalysis as within the disciplines making use of blood PK data.

\section{Considerations on venous versus} capillary (finger prick) blood sampling

The difference between venous versus capillary blood is not considered to be a bioanalytical property that needs investigation beyond the above mentioned hematocrit considerations. We acknowledged that analysis of venous blood versus capillary blood samples may impact the PK parameters. Evaluation of these differences is considered to be the responsibility of the pharmacokineticist.

\section{Additional considerations on physical blood parameters}

Although the scientific impact is not anticipated to be relevant for most drugs, the impact of hemolysis should be understood (i.e., on the spot formation and on sample stability). It is recommended to document the impact of hemolysis during method development. Observations made during method developement may initiate additional work in method development or validation.

The impact of other blood parameters affecting the robustness of DBS assays, such as lipidemic samples or other conditions, needs to be understood and validated as appropriate, similar to plasma/serum analysis.

\section{Considerations of the anticoagulant}

The principles around the use of anticoagulant for the preparation/spiking of validation samples and for study samples are currently considered as for plasma assays. However, because the DBS sample collection process differs from plasma samples, with a potential increased impact on clotting, additional sampling and spotting techniques may need to be developed and evaluated for this technique. If applicable, special considerations should be given to the technical challenges related to sampling microvolumes, such as undue sample dilution caused by addition of anticoagulants. The same considerations apply for additional additives such as stabilizing agents. It is recommended that all aspects of anticoagulant addition, including mimicking the sampling condition in real time, are managed during validation and throughout sampling and analysis. Being mindful of the potential technical or scientific hurdles when sampling blood on anticoagulant, the added value of using anticoagulants needs consideration. Additional considerations on sample dilutions above and beyond the ones caused by anticoagulants are discussed later.

\section{Incurred sample reanalysis}

Incurred sample reanalysis is applicable to DBS samples, as proposed in the relevant papers $[18,19]$. Depending on supporting validation data, ISR can be managed from the second spot for the same sample, or from a second spot punch taken out of the same spot.

\section{Superfluous validation experiments for DBS methods}

The outcome of the EBF-surveys was surprising in the sense that the EBF community did not identify any experiment to be superfluous when shifting from liquid assays to DBS assays, with the exception of those experiments specifically designed for stability assessment of samples in liquid (or frozen) state as referred to in chapter $\mathrm{D}$, paragraphs one to three of the CDER/FDA guidance [14]. As such, freeze-thaw experiments and short- or long-term stability assessment at different temperatures related to storage of liquid sample can be omitted in the validation experiments as they do not represent the DBS practices. The EBF identified a number of replacement experiments that will be discussed below to cover for the regulatory requirements relating to the introductory statement in the above mentioned CDER/FDA guidance [14] but replacing 'biological fluid' by 'sample matrix': "Drug stability in a biological fluid is a function of the storage conditions, the chemical properties of the drug, the matrix and the container system".

All other validation experiments typically performed as part of plasma/serum assay validation were identified as being relevant for DBS method validation.

\section{Validation experiments needing enhancements for DBS methods \\ - Sample homogeneity prior to \& during spotting}

Robustness and reproducibility of any bioanalytical assay is affected by the ability to take a representative aliquot from a homogenous sample. Microsampling technology applied in DBS may impact sample homogeneity to a major 
extent and therefore requires special consideration. Some examples of parameters that can negatively impact sample homogeneity of spiked samples are, time between spiking the analyte in blood and application of the spiked sample onto the card (e.g., stability aspects and equilibration after spiking); vigorous shaking of blood prior to spiking on card (e.g., introducing hemolysis); or application of multiple sample aliquots from one capillary versus from multiple capillaries. Both during method development and validation, and during the sampling steps of preclinical and clinical studies, it is desirable to provide proper guidance to the clinical and laboratory personnel to create awareness of the potential risks of improper sample handling.

\section{Preparation of CAL \& QC samples}

Preparation of spiked samples for DBS, such as $\mathrm{Cal} / \mathrm{QC}$ samples or stability samples, confronts the bioanalytical scientist with additional challenges compared with liquid-matrix samples, particularly when small sample volumes are used. As mentioned above, with DBS samples typically originating from microsampling procedures, these in vivo sampling procedures need to be developed and validated to prevent undue sample dilution with anticoagulants or stabilizing agents. Hence, when fortifying blood with analytes it is important to minimize the percentage of nonaqueous components added to prevent solvent effects creating differences between spiked versus incurred samples. Inappropriate attention to sample dilution aspects may induce differences in spot formation, distribution of the compound on the filter paper, hemolysis prior to applying to the filter paper or drying time. Also, incubation/equilibration dynamics of the analyte in fortified (spiked) samples versus incurred samples can differ and needs proper attention. Sample homogeneity may not be adequate when handling and mixing small sample volumes during fortifying steps.

Blood used to prepare Cal/QC samples should, where possible, be fresh (i.e., harvested on the day of use). If not routinely feasible, the effect of age (and storage) of the blood needs to be assessed (i.e., experiments or from literature) and documented. The use of batch-prepared Cal/QC samples is acceptable provided they are used within the documented storage stability period.

\section{Carryover}

Awareness of the potential for carryover from a number of sources should be considered during validation experiments and study sample analysis.
In addition, carryover originating from postpreparative and instrumentation carryover (e.g., autosampler and LC columns) should be considered. The following additional carryover considerations need to be properly assessed: physical carryover from card to card when stored together, and spot-to-spot carryover originating from the punching device. All confirmed sources of carryover should be eliminated (by inclusion of blank cards and/or punches in the run). Acceptance criteria should be the same as for liquid assays and managed in an SOP.

\section{Considerations for internal standards}

The bioanalytical community is of course very familiar with the concept of internal standards (IS). Nevertheless, we want to give special attention to the definition and the consequences for DBS samples. An IS is a close structural analogue of the analyte being quantified (preferably a stable isotope labeled analogue). The IS is added in equal concentration to all samples in an analytical run (to Cal/QC and study samples) to compensate for fluctuations in the analyte response during sample preparation and analysis. It is important to acknowledge that currently used procedures of adding the IS for DBS analysis, typically in solution in an organic solvent used to extract the DBS filter punch, does not cover all necessary aspects of analytical compensation intended by an IS. The IS will potentially not compensate for fluctuations in extraction or redissolving of the analyte of interest from the DBS filter punch. Additional scientific validation will be needed to investigate potential differences in behavior of the analyte (i.e., dissolving from the DBS filter punch) versus the IS (added in solution). The effects of extraction recovery differences may potentially increase due to longerterm storage. To date, insufficient data on longterm stability (multiple years) are available to fully understand the potential issues. As more data become available, the scientific community will gain more experience with this topic. In addition, other techniques of introducing the IS earlier in the analytical process should be considered and evaluated as appropriate.

\section{Extraction recovery}

The current FDA guidance is very specific on the requirements for extraction recovery, "...recovery of the analyte need not be 100\%, but the extent of recovery of an analyte and of the internal standard should be consistent, precise and reproducible. Recovery experiments should be 
performed by comparing the analytical results for extracted samples at three concentrations (low, medium and high) with unextracted standards that represent $100 \%$ recovery" [14]. Being mindful of the limitations of the analytical compensation offered by the IS with respect to extraction recovery and the potential differences with the analyte on the filter paper, recovery needs to be evaluated and documented more thoroughly for DBS assays.

The impact of card storage on extraction recovery from the punched disks needs to be considered and documented (i.e., as part of long-term stability). During method development and validation, consideration should be given to anticipate potential changes in extraction behavior of the analytes from old versus recent dried blood.

\section{Sample dilutions}

The principles of dilution used for liquid samples also apply for DBS. Scientific validation of dilutions should be documented during method validation. However, due to the specific limitations of the technique, a DBS sample cannot be diluted straightforward as for liquid matrix samples. One approach is to dilute the sample after the extraction of the punched disk with an extract of a blank DBS sample. Alternatives to the above dilution procedures with blank DBS extract, which may be costly or impractical and require a lot of blank spotted material, can be evaluated and subsequently validated. It should be understood that, when applying alternative approaches, the potential effect on other validation parameters (e.g., extract sample stability, matrix effect and selectivity) should be validated as part of the dilution testing.

\section{Matrix effects}

Similar to liquid assays, the guiding principles to document and/or prevent matrix effects apply for DBS assays. Although an integral part of the validation, awareness of the additional impact of the filter paper, especially pretreated filter paper, on matrix effects should be understood.

\section{Additional validation experiments needed for DBS methods}

- Filter paper consideration: full versus partial validation

The EBF recommends that a method needs to be fully validated per filter paper type/manufacturer. Any change in filter paper type and/or manufacturer requires a partial validation. Validation parameters to be considered for the partial validation initiated by this change in filter paper type and/or manufacturer need to be predefined in appropriate documentation. Recommended parameters included in this partial validation are: linearity and sample dilution, accuracy and precision, extraction recovery, matrix effects, drying conditions (i.e., drying time and temperature) and on-card storage stability. Additional parameters can be added, depending on the change induced by the change in filter paper.

\section{Good blood-spotting practices}

In order to ensure the quality of the blood spot and, hence, the reproducibility of the results obtained from DBS analysis, it is important that the application of the blood on the filter paper is executed by trained personnel aware of all the potential hurdles and issues of the technique. As such, the EBF reflected on the terminology to create awareness of the importance of accurate and reproducible spotting. For the purpose of this paper, we introduce the term good blood-spotting practices (GBSP) to connect the community with the importance of quality spotting with other GxP procedures. In fact, not adhering to GBSP will inevitably result in poor-quality samples and unreliable results.

Some key examples of not adhering to GBSP are spotting an incorrect volume (outside of the boundaries prescribed in the protocol or method validation report), incidental (partly) double spotting, applying spots too close to each other causing them to become confluent, applying spots close the edge of the filter paper or outside the preprinted area and dropping the spot onto the card surface. In addition, inappropriate manipulation of the filter paper (i.e., touching the filter paper with your fingers) prior to spotting or during and after drying can lead to contamination of the filter paper and should be avoided at all times.

The impact of a number of other potential poor-spotting practices is not fully recognized or understood yet, but additional care should be taken to avoid the application of spot in fractions versus one fluent movement or touching the filter paper with the capillary during spotting.

Other inappropriate spotting practices may be identified and should be shared with the scientific community as experience with the techniques grows. 


\section{Considerations for volume spotted and punched spot size}

The EBF recommends that the validation of the spot size, in millimetres punched from a DBS card, and originating from a predefined sample volume, is an integral part of the validation of a DBS method.

Many researchers have investigated and reported on the need to spike accurate samples, generally concluding that spiking accurate volumes is not necessary to obtain accurate DBS results [12]. Indeed, if accurate spiking of microvolumes of blood would be a prerequisite, this may pose a serious roadblock for the wide applicability of DBS in regulated bioanalysis, unless suitable apparatus can be developed that results in acceptable precision and bias. Validation data show that, when applied in an analytical setting, it is not necessary to know the accurate volume of the spiked spot as long as blood is homogeneously spread across the area that is punched and that an accurate sized punch is taken from the result in spot. Hence, a nominal sample volume should be stipulated as part of the method validation. Adherence to this volume should be easy to check by visual inspection of the dried spot. For example, the reproducibility of the assay is not affected when spotting volumes between 10 and $20 \mu$ for an assay that is validated for a volume of $15 \mu$ l, provided that a fixed sized punch is taken from the sample. However, it is not recommended to allow just any volume to be spotted onto a card, as this will lead to inappropriate spot formation, drying or sample handling. Consequently, EBF provides following guidance for the spot size validation and potential boundary testing needed as part of validation of the spot size.

If spotting on card is done using accurate pipettes (and documented in the study), no boundary testing is required as part of method validation. If spotting on card is done using nonaccurate tools (e.g., noncalibrated capillaries), boundary testing of spotted volume should be considered during validation in order to support using nonaccurate tools for spotting. As boundaries, typically a volume of $\pm 50 \%$ than the target volume would be appropriate. Acceptance criteria for boundary testing should be preset and aligned with acceptance criteria applied in regulated bioanalysis. Successful boundary testing should remove the need for accurate pipetting in the animal laboratory or clinic provided proper training to comply with GBSP is offered and spotting guidance is provided in the protocol, laboratory manual, SOP or equivalent. In all cases, target spot size for Cal/QC samples should be equal to that of the study samples.

Additional validation parameters related to the spot size are drying time, drying temperature (and definition thereof, i.e., 'ambient') and relative humidity (RH). As for the spot size, room temperatures and $\mathrm{RH}$ boundaries may need to be investigated as part of validation.

\section{Spot homogeneity}

As discussed broadly amongst scientist involved in DBS, spot homogeneity is a very important aspect of DBS bioanalysis. Spot homogeneity can be negatively influenced by a number of factors relating to the filter paper quality, physiological parameters of the blood sample and drying conditions to name a few. Although spot homogeneity usually does not seem to be an issue, the EBF recommends taking a big enough punch to be a representative sample (e.g., taking 3 -mm punches or lager from a $15-\mu$ ldried spot) and/or punch from the same location of a spot.

Multiple punches from the same spot

Analyzing multiple punches from the same spot can be needed for a number of reasons: increased sensitivity, enhanced accuracy and precision, reanalysis, ISR, additional metabolite quantification, biomarker analysis and so on.

Based on reflections and recommendations discussed in the definition of a sample and the above paragraph on spot homogeneity, the EBF recommends using the replicate spot over punching from the same spot, if this replicate spot is available. Nevertheless, the experiment may call for a second punch from an originally punched spot for additional analysis, or for multiple punches from one spot for first analysis. In these cases, and if absolute concentration values in $\mathrm{ng} / \mathrm{ml}$ are the intended outcome of the analysis, the EBF recommends only doing this if on-card sample homogeneity is assessed during validation or qualification of the method.

\section{Sample stability for DBS}

Short- and long-term stability of the sample is a key aspect in any bioanalytical method validation. Within the current regulations it is very clearly described how this should be managed. From the FDA guidance, "Stability procedures should evaluate the stability of the analytes during sample collection and handling, after long-term (frozen at the intended storage temperature) and 
short-term (bench top, room temperature) storage, and after going through freeze-thaw cycles and the analytical process. Conditions used in stability experiments should reflect situations likely to be encountered during actual sample handling and analysis" [14]. Going forward, and in line with evolving science and emerging regulations, the bioanalytical community is anticipating further refinements for stability assessments.

Over the last decade, the industry has been developing experimental protocols to assess all stability aspects that all focus on the stability for liquid samples at room temperature or in frozen conditions. For DBS, the same principles will be applicable. However, a considerable rethink is needed to address the specific needs of dried samples. It is important to note that there are multiple examples reported on significantly enhanced stability when using DBS compared with traditional liquid assays, especially in the area where compound instability is caused by esterase activity. In fact, tackling stability issues is certainly an area where DBS has already proven its added value and will be of critical use in the future. Nevertheless, being mindful that the technique is still relatively new in the area of regulated bioanalysis, some caution is warranted and the following recommendations are put forward by EBF.

\section{- General}

Evaluation of stability aspects mentioned in the current regulatory guidances, but which are scientifically or procedurally irrelevant for DBS should be discontinued (i.e., freeze-thaw).

\section{- Recommendations for blood stability} prior to spotting

In addition to investigation of pre- and postanalysis on-card stability, appropriate stability experiments should be conducted on liquid whole blood to be able to provide proper guidance to the collection and handling of blood samples prior to spotting. The blood used for these experiments should be freshly harvested and the experiments should be performed at body temperature $\left(37^{\circ} \mathrm{C}\right)$ to reflect the in real-life situation.

\section{- Recommendations for storage of DBS} cards prior to spotting

Recommendations for assessing stability of DBS cards prior to spotting should focus on storage and handling or manipulation or the cards. Prior to use, the cards need to be shipped to the sampling site and stored until use. Particularly for the DBS cards used in support of clinical trials in developing countries, storage of prespotted cards may occur under extreme environmental conditions (i.e., temperature/RH), which can negatively affect their performance. As a consequence, the EBF recommends shipping and storing cards under dry (desiccant) conditions prior to use. Equal precautions should be taken when manipulating the prespotted cards prior to use. As mentioned under 'GBSP', touching the surface of the filter paper can negatively influence the performance of the cards or induce contaminations.

\section{- Recommendations for storage of DBS} cards post-spotting

Once the blood sample is applied on the card, dedicated stability experiments need to be executed to understand the short- and long-term stability of DBS samples under the intended storage conditions. As for the prespotted cards, extreme environmental storage conditions at the sampling site (already potentially influencing drying conditions), during shipment and at the bioanalytical site, should be understood and appropriately validated. Examples include, but are not limited to: high RH, extreme room temperatures, temperatures and $\mathrm{RH}$ during shipment, sudden temperature/RH changes impacting condensation, extreme and long exposure to daylight. Boundary testing of mentioned parameters should be considered as part of the stability experiments during validation. Consequently, appropriate measures should be taken to prevent extreme environmental conditions during storage or shipment, such as desiccants and protection from light/extreme temperatures.

\section{Conclusion}

The EBF has intensively discussed the validation requirements needed to generate robust and reproducible concentration data from DBS experiments. We carefully identified and evaluated the validation requirements needed in addition or in excess of the method validation practices established for liquid assays. As such, numerous adaptations, enhancements or refinements are recognized to be essential to include for DBS method validation. With the experience of the technique growing, we identified some areas to which the industry needs to pay special attention when further developing the experience with the technique. The 
EBF intends to continue its connection with the bioanalytical community to share the successes and benefits of the technique, but also to bring the current identified hurdles to a resolution in support of the increased use of DBS in preclinical and clinical development.

\section{Financial \& competing interests disclosure}

The authors have no relevant affiliations or financial involvement with any organization or entity with a financial interest in or financial conflict with the subject matter or materials discussed in the manuscript. This includes employment, consultancies, honoraria, stock ownership or options, expert testimony, grants or patents received or pending, or royalties.

No writing assistance was utilized in the production of this manuscript.
Contributors, other European Bioanalysis Forum member companies:

Peter van Amsterdam, Abbott Stephanie Fischmann, Abbott Carl-Johan Sennbro, Active Biotech Josep M. Jansat, Laboratorios Almirall SA Hans Mulder, Astellas

Elizabeth Thomas, AstraZeneca

Margarete Brudny-Kloeppel, Bayer Schering Pharma AG

Magnus Knutsson, Ferring Pharmaceuticals

Klaus Pusecker, Grünenthal $\mathrm{GmbH}$

Berthold Lausecker, F. Hoffmann-La Roche

Morten Anders Kall H. Lundbeck A/S

Nathalie Mokrzycki, MSD

Marcel de Zwart, MSD

Achim Freisleben, Merck Serono

Fernando Romero, Novartis Pharma AG

Michael Pilgård Andersen, Novo Nordisk

Sirpa Laakso, Orion Corporation Orion Pharma

Richard Hucker, Pfizer

Dietmar Schmidt, Sanofi-Aventis

Ben Gordon, Servier

Richard Abbott, Shire Pharmaceuticals

Pierre Boulanger, UCB Pharma

\section{Bibliography}

1 Guthrie R, Susi A. A simple phenylalanine method for detecting phenylketonuria in large populations of newborn infants. A simple phenylalanine method for detecting phenylketonuria in large populations of newborn infants. Pediatrics 32, 338-343 (1963).

2 Edelbroek PM, van der Heijden J, Stolk LML. Dried blood spot methods in therapeutic drug monitoring: methods, assays and pitfalls. Ther. Drug Monit. 31(3), 327-336 (2009)

3 Beaudette P, Bateman KP. Discovery stage pharmacokinetics using dried blood spots. J. Chromatog. B 809, 153-158 (2004).

4 Li W, Tse FLS. Dried blood spot sampling in combination with LC-MS/MS for quantitative analysis of small molecules. Biomed. Chromatogr. 24(1), 49-65 (2010)

5 Wong P, Pham R, Bruenner B, James C. Increasing efficiency for dried blood spot analysis: prospects for automation and simplified sample analysis. Bioanalysis 2(11), 1787-1789 (2010).

6 Clark GT, Haynes JJ, Bayliss MAJ, Burrows L. Utilization of DBS within drug discovery: development of a serial microsampling pharmacokinetic study in mice. Bioanalysis 2, 1477-1488 (2010).

7 Barfield M, Spooner N, Lad R, Parry S, Fowles S. Application of dried blood spots combined with HPLC-MS/MS for the quantification of acetaminophen in toxicokinetic studies. J. Chromatogr. B Analyt. Technol. Biomed. Life Sci. 870, 32-37 (2008).

8 Patel P, Mulla H, Tanna S, Pandya H. Facilitating pharmacokinetic studies in children: a new use of dried blood spots. Arch. Dis. Child. 95, 484-487 (2010).

9 Corran P, Cook J, Lynch C et al. Dried blood spots as a source of anti-malarial antibodies for epidemiological studies. Malaria J. 7, 195 (2008).

10 Solomon S, Solomon S, Rodriguez I et al. Dried blood spots: a valuable tool for HIV surveillance in developing/tropical countries. Int. J. STD AIDS 13, 25-28 (2002).

11 Heinig K, Wirz T, Bucheli F, Gajate-Perez A. Determination of oseltamivir (Tamiflu $\left.{ }^{\circledR}\right)$ and oseltamivir carboxylate in dried blood spots using offline or online extraction. Bioanalysis 3(4), 421-37 (2011).

12 Spooner N, Lad R, Barfield M. Dried blood spots as a sample collection technique for the determination of pharmacokinetics in clinical studies: considerations for the validation of a quantitative bioanalytical method. Anal. Chem. 81(4), 1557-1563 (2009).

13 Abbott R, Smeraglia J, White S et al. European Bioanalysis Forum Workshop: connecting strategies on dried blood spots. Workshop report. Bioanalysis 2(11), 1809-1816 (2010).

14 Guidance for Industry Bioanalytical Method Validation, US Department of Health and Human Services Food and Drug Administration Center for Drug Evaluation and Research (CDER), Center for Veterinary Medicine (CVM), May 2001.

15 Viswanathan C, Bansal S, Booth B et al. Workshop/Conference Report - quantitative bioanalytical methods validation and implementation: best practices for chromatographic and ligand binding assays. Workshop/Conference Report. AAPS J. 9(1), E30-E42 (2007).

16 Rowland M, Emmons G. Use of dried blood spots in drug development: pharmacokinetic considerations. AAPS J. 12, 290 -293 (2010).

17 Emmons G, Rowland M. Pharmacokinetic considerations as to when to use dried blood spot sampling. Bioanalysis 2, 1791-1796 (2010).

18 Fast D, Kelley M, Viswanathan CT et al. Workshop report and follow-up - AAPS Workshop on Current Topics in GLP Bioanalysis: assay reproducibility for incurred samples - implications of Crystal City Recommendations. AAPS J. 11(2), 238-241 (2009)

19 Timmerman P, Luedtke S, van Amsterdam P et al. Incurred sample reproducibility: views and recommendations by the European Bioanalysis Forum, Bioanalysis 1(6), 1049-1056 (2009)

\section{- Websites}

101 PSWC/AAPS meeting. New Orleans, USA, November 2011. www.pswc2010.org

102 EBF meeting. Barcelona, Spain, December 2011. http://bcn2010.europeanbioanalysisforum. eu/slides

103 Global Bioanalysis Consortium website. www.globalbioanalysisconsortium.org 